\title{
The Optical Fibre Internet: Where Next?
}

\author{
David N Payne, CBE FRS FREng FIET FInstP \\ Director \\ Optoelectronics Research Centre \\ University of Southampton
}

\begin{abstract}
Powering the optical fibre internet with its huge global reach, photonics has changed our lives. Optical fibres snake across continents and oceans carrying terabits per second of data in a vast information network that brings untold human connectivity. But capacity demand continues to grow at a startling rate, doubling every two years, while the internet is estimated as burning several percent of world energy usage. The optical internet is reaching its capacity limits. The solution to these consequences of unbridled demand is more photonics, reaching further into the network with optics to overcome the existing bottlenecks and employing next-generation optical components - Internet 2.0.

The great success of optical fibres and planar circuits in telecommunications has generated numerous tantalising applications in a number of related fields, such as sensing, bio- and nano-photonics and high-power lasers. Incredibly, the same fibres that carry tiny internet signals can also generate kilowatts of power, sufficient to cut through inch-thick steel and perhaps drive the next generation of high energy physics.

The talk will celebrate optical fibre technology and asks what next?
\end{abstract}

\section{Short Bio}

David Payne is Professor of Photonics and Director of the Optoelectronics Research Centre (ORC) at the University of Southampton. He has published over 650 Conference and Journal papers and is co-inventor on over 40 patents. Over the last forty years, he has made several key contributions in optical fibre communications and laser technology. His work in fibre fabrication in the 1970 s resulted in most of the special fibres used today, including the revolutionary erbium-doped fibre amplifier (EDFA) and kilowatt-class fibre lasers for manufacturing and defence. He has received the UK Rank Prize for Optics, the 2001 Mountbatten Medal, the 2004 Kelvin Medal for the application of science to engineering, the 2007 IEEE Photonics Award, the 1991 IEEE/LEOS Tyndall Award, the 1998 Benjamin Franklin Medal for Engineering, and is Laureate of the 2008 Millennium Technology Prize. He is

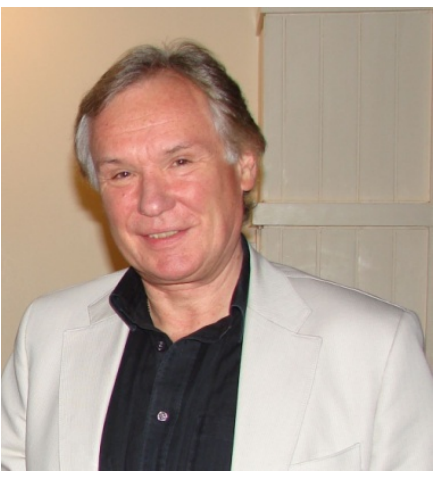
also an Eduard Rhein Laureate and a foreign member of the Norwegian and the Russian Academies of Sciences. He is a Fellow of the Royal Society, the Royal Academy of Engineering, the Optical Society of America, the IET and the IoP. As an entrepreneur, he founded York Technologies, (now PK Technology Inc.) and SPI Lasers plc (now part of the Trumpf Gruppe). 